\title{
Visión holística sobre el teletrabajo
}

\section{Katherin Santillán Guzmán}

katherin.santillan@upeu.edu.pe

ORCID: 0000-0002-6623-7727

Escuela de administración y negocios internacionales

Universidad Peruana Unión

\section{Renán Saavedra Sandoval}

rsaavedrasa@unmsm.edu.pe

ORCID: $\underline{\text { 0000-0002-3018-9460 }}$

Escuela de posgrado

Universidad Nacional Mayor de San Marcos

Tarapoto - Perú

\section{RESUMEN}

El presente estudio de revisión tiene como objetivo principal, indagar sobre las características, elementos básicos e implicancias del teletrabajo. El tipo es cualitativo y el diseño de estudio es una revisión sistemática; asimismo, se entiende que el teletrabajo es la modalidad del trabajo a distancia prestado mediante el uso de las telecomunicaciones; también, se afirma que es la realización del trabajo desde el domicilio; sin embargo, no necesariamente ha de ser así, pues la actividad laboral puede entenderse siempre que sea ejecutada desde diferentes entornos y/o lugares ajenos a la empresa o institución. Los principales resultados de la presente investigación son: a. El teletrabajo es una labor a distancia con el uso de las TIC's, b. El teletrabajo puede ser una buena oportunidad, en el mundo globalizado en el que vivimos, c. El teletrabajo es una opción laboral que aún falta regular, d. A nivel nacional, no existe mucho entusiasmo, por las carencias existentes. El estudio concluye, que: Un 50\% de investigadores afirman que implementar el teletrabajo, requiere de la empatía entre empleado y empleador. Asimismo, el 30\% de investigadores afirma que es urgente la regulación del teletrabajo. De igual forma, el $75 \%$ de los encuestados nacionales cree que, para la debida implementación del teletrabajo, debe previamente garantizarse, los espacios, herramientas tecnológicas, manuales y regulaciones correspondientes.

Palabras clave: teletrabajo; trabajo a distancia; trabajo en red; tecnologías; holístico. 


\title{
Holistic visión of teleworking
}

\begin{abstract}
The main objective of this review study is to investigate the characteristics, basic elements, and implications of telework. The type is qualitative and the study design is a systematic review; Likewise, it is understood that telework is the modality of distance work provided through the use of telecommunications; also, it is affirmed that it is the realization of work from home; However, this does not necessarily have to be the case, since the work activity can be understood as long as it is carried out from different environments and / or places outside the company or institution. The main results of this research are: a. Teleworking is remote work with the use of ICTs, b. Teleworking can be a good opportunity, in the globalized world in which we live, c. Teleworking is a job option that is still lacking in regularity, d. At the national level, there is not much enthusiasm, due to the existing deficiencies. The study concludes that: $50 \%$ of researchers affirm that implementing telework requires empathy between employee and employer. Likewise, $30 \%$ of researchers affirm that the regulation of teleworking is urgent. Similarly, $75 \%$ of national respondents believe that, for the proper implementation of telework, the spaces, technological tools, manuals, and corresponding regulations must be previously guaranteed.
\end{abstract}

Keywords: telework, remote working, networking, technologies, holistic

Artículo recibido: 05 octubre. 2021 Aceptado para publicación: 02 noviembre 2021 Correspondencia: katherin.santillan@upeu.edu.pe Conflictos de Interés: Ninguna que declarar 


\section{INTRODUCCIÓN}

Una visión holística del teletrabajo, es una revisión somera e integral, pero a la vez diversa y abierta de esta nueva modalidad laboral llamada teletrabajo; es decir, el presente estudio, pretende diagnosticar la realidad del teletrabajo, especialmente en nuestro país, de manera integral, enfocándola desde diversos puntos de vista como son: sus conceptos, modalidades, ventajas y desventajas, efectos, regulaciones, empatías entre empleador y colaborador, entre otros.

Hablar del teletrabajo en tiempos actuales, equivale a dialogar, debatir y analizar la noción y componentes, de una nueva modalidad laboral, que es sumamente amplia; por lo mismo, existen definiciones diversas al respecto; nuestro trabajo pretende investigar, comparar y analizar dichas definiciones y toda la realidad problemática y las posibilidades del teletrabajo, en los contextos correspondientes, en el ámbito internacional, nacional y regional; en coherencia con lo indicado queremos iniciar este apasionado proyecto, presentando algunas de las definiciones halladas al respecto:

El teletrabajo es una modalidad laboral, que puede realizarse bajo un acuerdo predeterminado entre el empleador y sus colaboradores, el cual puede realizarse desde el domicilio u otro lugar distante al centro laboral presencial y haciendo uso de las tecnologías de la información y la comunicación disponibles (Sanguinetti, 2017)

El teletrabajo es una forma laboral, para la ejecución de un trabajo, a partir de un contrato entre las partes, usando los medios como las tecnologías informáticas y otros medios necesarios para tal realización, (Saco, 2007)

EI teletrabajo es una labor realizada por una persona, desde su domicilio, u otro lugar acondicionado, haciendo uso de las tecnologías de la información y la comunicación, además de otros medios necesarios. (Raso 2017)

EI teletrabajo es entendido, como la forma de la realización de las diversas actividades profesionales con el uso de la informática, el procesamiento de la información y dándole el valor agregado a la misma. (Gonzales, 2007)

EI teletrabajo es una forma laboral, en la que las personas desarrollan sus funciones fuera de las oficinas o centro laboral presencial, sin mantener contacto con sus 
compañeros de trabajo, pero que sí pueden comunicarse con ellos, por medio de las nuevas tecnologías. (Wirth, 2004)

En el Perú el teletrabajo, actualmente, es un tema de confianza entre el empleador y el colaborador; pasa por respetar tiempos y jornadas laborales; con el trabajo asincrónico, las urgencias se enfocan, en la creación de normas para el teletrabajo, llenar los vacíos legales existentes; mientras ello sucede, las claves deben girar alrededor de la empatía; en suma, mientras se termine de reglamentar, el tema estará siempre, en las voluntades, los detalles y gestos de las partes. (Carrillo, 2020)

Asimismo, en el Perú, la debida implementación del teletrabajo, pasa aún por realizar un verdadero análisis, para conocer todas sus implicancias y establecer los niveles de satisfacción que generan al empleador, pero también el respeto, las comodidades y satisfacciones, que debe establecerse para con el colaborador o trabajador virtual.

Por nuestra parte, sintetizando, los conceptos de teletrabajo, podemos afirmar, que es una forma laboral alejado de las oficinas; es una labor altamente especializada, sin contacto físico con los demás colaboradores y usando diversos medios tele informáticos; es decir, actividad que se realiza, en un lugar distinto y distante al centro laboral.

En coherencia con la realidad descrita, líneas arriba, la presente investigación, se plantea como objetivo principal, la revisión metódica y sistemática de la información disponible sobre el teletrabajo, para caracterizarlo, valorar sus implicancias prácticas y su aporte en el desarrollo social; asimismo, sugerir subsanar sus deficiencias, y plantear nuevas temáticas de desarrollo, para proporcionar las herramientas y estrategias adecuadas, a fin de lograr para el futuro, un teletrabajo más eficiente que el actual.

\section{REVISIÓN GENERAL}

En el desarrollo del proceso de investigación, consideramos como primera fase o etapa la revisión general de información valiosa sobre la materia.

\subsection{Aspectos jurídicos del teletrabajo.}

Thibault (2019), nos dice que: cuando en el panorama socio laboral surge un fenómeno nuevo, como el teletrabajo, la primera tarea que debe afrontar el jurista laboralista es la de determinar la naturaleza jurídica, incluyéndolo en alguna de las categorías legales ya existentes, o en caso de imposibilidad, reclamar una regulación ad hoc. 
Esto consiste en resolver, si el teletrabajo supone una forma de trabajar que estaría enmarcada dentro de una relación laboral o una forma autónoma, lo que se entendería que se trata de una relación civil, es decir, se trata de dilucidar si la relación de teletrabajo tiene carácter laboral o civil. Por las consideraciones indicadas, no resulta tan claro cuando se está ante una u otra situación, ya que el hecho de que tenga que entenderse que sea una relación civil o una relación laboral, dependerá de las diferentes modalidades de cómo se lleve a cabo o se ejecute el mismo.

Asimismo, Sellas (2001), sostiene que: el teletrabajo puede ser calificado como una prestación de servicio sometida al ámbito de aplicación del Derecho Mercantil, y dentro del mismo, encuadrado como trabajo por cuenta propia o autónomo, o al ámbito del Derecho del Trabajo, y dentro de éste, encuadrado como trabajo por cuenta ajena o subordinada.

La prenombrada autora nos indica con ello que, para que el teletrabajador esté encuadrado o considerado como trabajador por cuenta propia o autónomo, se requiere que el mismo tenga la facultad de disponer de plena autonomía en la organización de la actividad económica, con la consiguiente reversión en beneficio propio de la utilidad patrimonial resultante de dicha actividad económica, así como la asunción del riesgo derivado de su ejecución. Asimismo, nos dice, que no basta con cumplir lo antes indicado, debe concurrir a la vez los cuatro requisitos objetivos siguientes: el ánimo lucrativo con que debe ejercitarse la actividad económica; la habitualidad con que ha de desarrollarse; la intervención directa y personal en la misma, y la inexistencia de contrato de trabajo.

\subsection{Regulación del teletrabajo en el Perú.}

El teletrabajo en el Perú se encuentra regulado, a través de la Ley $\boldsymbol{N}^{\circ} \mathbf{3 0 0 3 6}$, Ley que regula el Teletrabajo y su Decreto Supremo No 017-2015-TR, que aprueba el Reglamento de la Ley $\mathrm{N}^{\circ} 30036$, Ley que fue aprobada por los Proyectos de Ley $\mathrm{N}^{\circ}$ 184/2011 y 1052/2011. Lo que demuestra que no fue viable proponer una positivización del teletrabajo, sin antes realizar todo un análisis para determinar y demostrar que existen motivos que ameritan, la regulación y aplicación de esta nueva figura en nuestra realidad socio laboral, tal y como lo demuestran las exposiciones de motivos de cada uno de los proyectos de ley que sustentan la hoy promulgada Ley $\mathrm{N}^{\circ} 30036$, Ley que regula el Teletrabajo en el Perú. 


\subsection{Proceso histórico del teletrabajo en el Perú.}

En el Perú, se empezó a hablar del teletrabajo en el 2011, año en el que se comenzó a debatir el proyecto de ley; en el 2013 se dieron facultades a las empresas, para contratar, bajo esta modalidad a las personas con habilidades diferentes, eliminando así las barreras físicas y de espacios, más aún, si se tenía en aquel entonces, la renuencia de las empresas a contratar a personas con habilidades diferentes, quizá por no contar con espacios adecuados para las labores de dichas personas, por lo que el teletrabajo se convertía en la mejor opción, pues traería como ventaja mayor comodidad para el teletrabajador y la disminución del ausentismo y riesgos dentro del centro laboral. Todo ello hacía impostergable la necesidad de regular la figura del teletrabajo que conjugaba, en ese entonces, con la flexibilidad de la seguridad laboral, caso contrario se corría el riesgo de desvalorizar toda relación de trabajo.

En ese contexto, en el año 2015, con la declaración de la Ley $\mathrm{N}^{\mathrm{o}} 30036$, Ley del Teletrabajo, se pretendió que el empresario acceda a una nueva forma de trabajo no presencial, permitiendo generar los mismos ingresos, pero abaratando ciertos costos, beneficiando, también, al teletrabajador; sin embargo, la figura jurídica como tal, ha sufrido una resistencia en el Perú, quizá porque faltan algunas herramientas de promoción, aplicación e implementación para el teletrabajo. Lo que ha conllevado a que sea una ley casi invisible, pero que sí es utilizado por un aproximado del $5.6 \%$ del empresariado.

Asimismo, en noviembre del 2016, el Ministerio de Trabajo y Promoción del Empleo firmaron acuerdos con los gobiernos regionales, empresas y otros organismos, sobre el teletrabajo, con la finalidad de implementar el teletrabajo en el sector público y privado, sin embargo, lo que observamos en la realidad, es que solo fue un simple pacto, sin que llegue a concretizarse, pues a pesar de ello, la figura jurídica del teletrabajo, si bien es cierto era refrendada a través de la Ley $\mathrm{N}^{\circ}$ 30036; también es cierto que el teletrabajo no era la figura protagonista dentro de un mundo tecnológico de vanguardia; por el contrario, a pesar de la creación e implementación de Módulos de Teletrabajo en las regiones de Tacna, La Libertad, Piura, Cusco y Callao, sin embargo, no se logró la proyección esperada para la utilidad de la Ley $\mathrm{N}^{\mathrm{o}} 30036$, por lo que se entiende que el teletrabajo se ha tratado de una figura casi fantasma, que no ha sido utilizada en su totalidad por el empresario para obtener beneficios y aminorar costos. Precisamos que las pocas empresas 
que han venido utilizando desde el año 2014 los teletrabajos son: Laboratorios Roche, Repsol, entre otras. Correa (2019)

Finalmente, en el año 2020, a pesar, de que el teletrabajo en el Perú cuenta con normatividad expresa (Ley $\mathrm{N}^{\circ}$ 30036) que regula su aplicación, en los tiempos que vivimos de confinamiento social a consecuencia del COVID-19, no es utilizada o no ha sido llamada para su aplicación por el empresariado; muy por el contrario, a través del Decreto de Urgencia $\mathrm{N}^{\circ}$ 026-2020 (15.03.2020), que establece ciertas medidas para prevenir y combatir el COVID-19, expresa en su artículo $17^{\circ}$ la aplicación del "trabajo remoto" (figura distinta al teletrabajo, pero sí una modalidad o derivación dinámica del mismo), que es entendido, según la norma actual, como la labor realizada desde el domicilio y no desde la oficina, estableciéndose además, su aplicación en el sector público y privado.

\subsection{Teletrabajo durante la pandemia.}

Cuando hablamos del teletrabajo durante la pandemia, empezaremos indicando, que ésta nació por una extrema necesidad, de tal manera que la gran mayoría de organizaciones u empresas la implementaron sobre la marcha y con premura; evidenciándose luego de algunos meses, las deficiencias, dificultades y carencias de una implementación repentina y sin criterio técnico.

Por otra parte, destacaremos, que antes de la aparición del COVID-19, no sólo a nivel nacional, sino que, en el mundo entero, la aplicación del teletrabajo, venía en un proceso insipiente, muy lejano de la realidad actual; tal es así que sólo el 10\% de las empresas en el mundo, podrían ofrecer a sus colaboradores la alternancia del teletrabajo, asimismo de cada tres personas, uno tenía idea del teletrabajo en su domicilio.

Finalmente indicamos que el teletrabajo se generaliza y masifica a razón de una gran necesidad de no paralizar en absoluto el funcionamiento estatal o empresarial, el cual se implementa con la mayor rapidez posible, para responder los desafíos que nos obliga el confinamiento por la lucha contra el coronavirus; lo cual sugiere que tendrá siempre deficiencias y dificultades; muy distinto hubieran sido los resultados si su implementación no se realizaba por necesidad, obligación u orden imperativo, sino por iniciativas propias sugeridas de los estados o empresas

\subsection{Adaptación del teletrabajo durante la pandemia.}

Por la naturaleza del ser humano y por las carencias, no será fácil para todos la adaptación al teletrabajo, porque no todos poseen las condiciones adecuadas, ni las herramientas 
necesarias; como tampoco cuentan con los espacios requeridos ni las habilidades exigidas, sin embargo, reconocer también, que con voluntad y decisión, el ser humano siempre es capaz para superar las dificultades, y resolver los problemas.

Por otra parte, destacamos que el organismo denominado Políticas Públicas para la Equidad y el Crecimiento (CIPECC), en uno de sus últimos estudios realizados, concluye que, un $40 \%$ de las actividades del ser humano exigen su realización presencial, y es casi imposible llevarlas a cabo, de manera remota o a través del teletrabajo, lo que significa un porcentaje alto de trabajo en la que sí es indispensable, la acción directa del hombre.

Asimismo, es sorprendente observar que hasta el inicio del 2020, todos los trabajadores se manejaban al menos dentro de tres entornos; el entorno profesional/laboral, el entorno social y el entorno familiar/íntimo; esos tres entornos, pocos meses después quedaron reducidos a tan sólo un espacio familiar pero que ya no es íntimo; y esta nueva realidad, no es de fácil adaptación para nadie, seguro causa y seguirá causando dificultades a todos y sólo el tiempo dirá si volveremos a recuperar a plenitud, los tres espacios o entornos que teníamos antes del inicio de la pandemia COVID-19

\subsection{Jornada laboral del teletrabajo durante la pandemia.}

Según un estudio de la universidad de Harvard y la universidad de New York, en un ámbito aproximadamente de 31 millones de persona, correspondientes a 21 mil empresas o compañías, establecen como conclusión principal que, durante el lapso de la cuarentena a razón de la pandemia COVID-19, en promedio, los colaboradores trabajaron 16 horas más semanalmente, lo cual constituye un abuso condenable y lamentable.

Por otra parte, en el lejano oriente, la realidad no es muy distinta, ya que se conocen que estudios realizados en China, concluyen que en promedio, durante la cuarentena impuesta por los gobiernos, los colaboradores pasaron de trabajar seis días a la semana, a laborar los siete días semanales, generando malestares y protestas de los ciudadanos; ambos estudios demuestran, que tanto en occidente como en oriente, ha habido y seguro que seguirá habiendo un aprovechamiento, abusivo en la carga laboral hacia el colaborador, so pretexto del trabajo remoto y la cuarentena por el COVID-19.

\subsection{Ventajas y desventajas del teletrabajo.}

El teletrabajo ofrece ventajas para la empresa y el teletrabajador; pero, al mismo tiempo, supone también desventajas, tanto para la empresa como para el colaborador. 


\section{Tabla 1}

Factores para la empresa.

\begin{tabular}{|c|c|}
\hline Ventajas & Desventajas \\
\hline $\begin{array}{l}\text { - El teletrabajo, supone un ahorro para la } \\
\text { empresa, porque evita pagos por } \\
\text { alquileres y gastos de oficina. } \\
\text { - El teletrabajo, a decir de los expertos, } \\
\text { significa una mejora en la } \\
\text { productividad del colaborador. } \\
\text { - Asimismo, el teletrabajo significa } \\
\text { también una flexibilidad en el contrato } \\
\text { de los colaboradores, especialmente, si } \\
\text { el colaborador es independiente. }\end{array}$ & $\begin{array}{l}\text { - Una desventaja principal del } \\
\text { teletrabajo, es su falta de regulación y } \\
\text { precisión, de sus alcances y } \\
\text { posibilidades. } \\
\text { - Otra desventaja para la empresa es que } \\
\text { existe, desconfianza y cierta } \\
\text { resistencia, para buena parte de los } \\
\text { colaboradores. } \\
\text { - También es una desventaja para la } \\
\text { empresa que, en la implementación de } \\
\text { la forma laboral del teletrabajo, } \\
\text { disminuye la seguridad y privacidad de } \\
\text { la información. }\end{array}$ \\
\hline
\end{tabular}

\section{Tabla 2}

Factores para el teletrabajador.

\begin{tabular}{|c|c|}
\hline Ventajas & Desventajas \\
\hline $\begin{array}{l}\text { - El teletrabajo, es ventajoso para el } \\
\text { teletrabajador, porque le ofrece mayor } \\
\text { autonomía e independencia en el } \\
\text { cumplimiento de sus funciones. } \\
\text { - También el teletrabajo, le ofrece al } \\
\text { teletrabajador una mayor flexibilidad } \\
\text { en los tiempos y horarios laborales. } \\
\text { - De igual forma el teletrabajo significa } \\
\text { una nueva oportunidad para el } \\
\text { teletrabajador, en tiempos en que el } \\
\text { trabajo es sumamente escaso. }\end{array}$ & $\begin{array}{l}\text { - Supone una gran desventaja para el } \\
\text { teletrabajador, recarga de horas, que } \\
\text { algunos empleadores, le asignan } \\
\text { aprovechándose del trabajo a distancia. } \\
\text { - Otra desventaja para el teletrabajador } \\
\text { es que no necesariamente cuenta con el } \\
\text { ambiente ni las herramientas } \\
\text { adecuadas; dificultando así su } \\
\text { desempeño. } \\
\text { - También supone una desventaja para el } \\
\text { teletrabajador, la exposición a muchas } \\
\text { horas y la exigencia mental y visual } \\
\text { frente a la PC o laptop, afectando de } \\
\text { esta manera su salud. }\end{array}$ \\
\hline
\end{tabular}

\subsection{Claves para un buen funcionamiento del teletrabajo.}

El teletrabajo puede ser una buena modalidad y una buena oportunidad laboral, siempre y cuando se viabilice y facilite su aplicación; sin embargo, todo, ello pasa, porque ambas 
partes tanto empleador como colaborador, estén plenamente de acuerdo, para la implementación y ejecución de dicha modalidad laboral, a partir del cual, el empleador deberá ofrecer todas las condiciones y herramientas necesarias, así como el colaborador, deberá ofrecer responsabilidad, voluntad para superar las dificultades y esfuerzo para alcanzar la eficiencia y eficacia laboral; es por ello que aquí presentamos algunas claves para el debido funcionamiento del teletrabajo.

\section{Tabla 3}

10 claves para que el teletrabajo funcione.

1. Considerarlo un trabajo tan serio como el presencial: Es necesario que el teletrabajador comprenda la seriedad del trabajo remoto, ya que equivale al presencial.

2. Marcarse horarios: El teletrabajador necesita una autodisciplina para establecer y cumplir estrictamente sus horarios.

3. Contar con espacio propio para trabajar: El teletrabajo merece un espacio y ambiente propios, adecuados para alcanzar la concentración plena.

4. Separar el ocio de las obligaciones laborales: Debe establecer la diferencia entre sus funciones laborales y compromisos sociales.
5. No llevar una vida sedentaria: El teletrabajo necesita de realizar ejercicios físicos, para poder rendir a plenitud y evitar enfermedades.

6. Realizar pequeñas pausas activas: Es recomendable que cada dos horas como máximo, realizar una pausa activa de 5 a 10 minutos.

7. Mantenerse en contacto son sus compañeros: Es muy importante la interrelación y el compartir información con sus pares.

8. Contar con los equipos necesarios: Es importante el poder tener un teléfono corporativo, un correo, laptop

9. No consultar redes sociales.

10. Contar con el apoyo de quienes viven con nosotros.

\section{REVISIÓN DE ARTÍCULOS CIENTÍFICOS}

Luego de haber culminado la revisión de la información general, sobre la presente investigación, como segunda fase se realizó, la revisión de artículos científicos; de los cuales presentamos los aportes y conclusiones, de los cuatro más importantes: 
Tabla 4

Investigaciones del teletrabajo

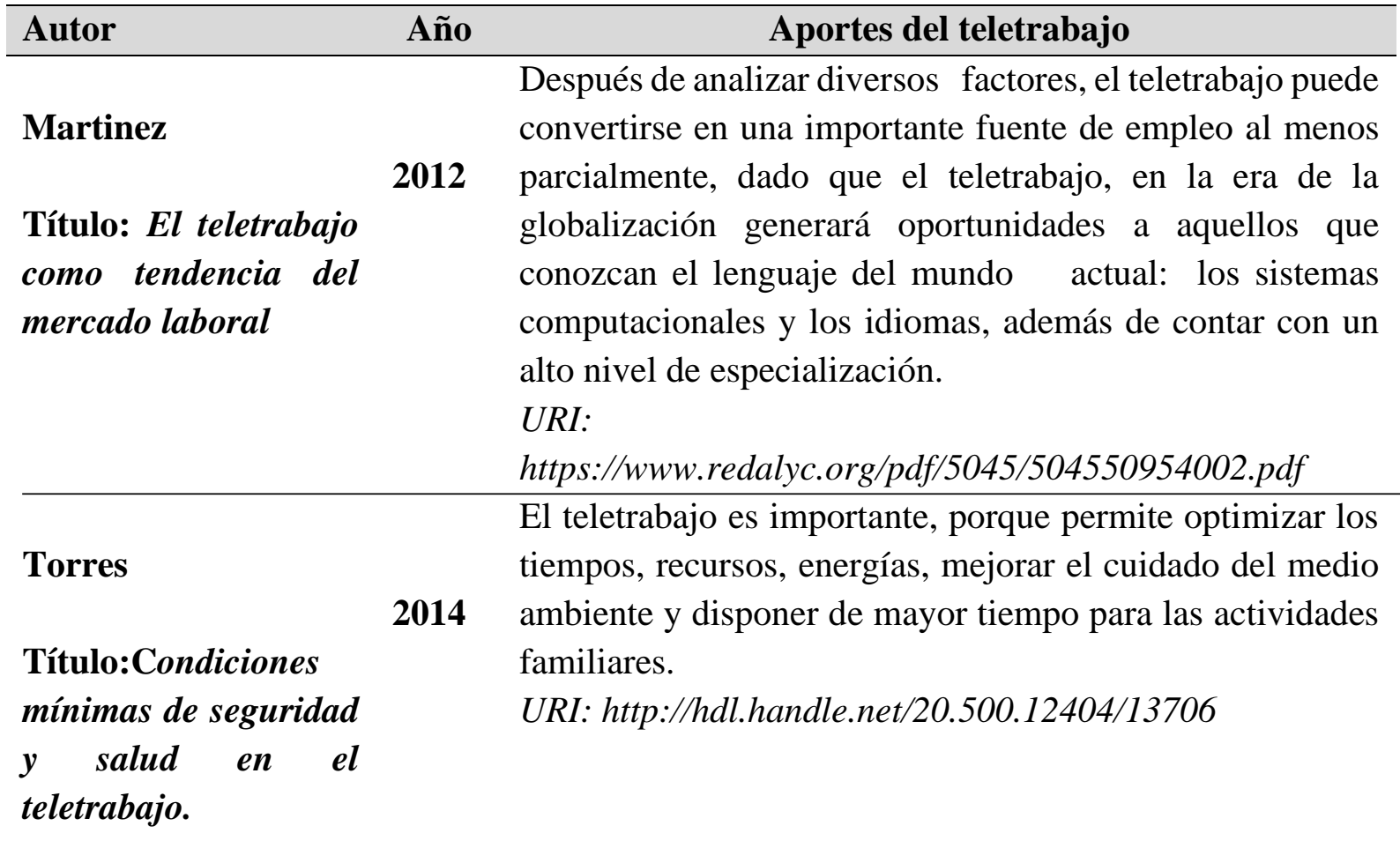

\section{Caminos}

Título: Análisis del teletrabajo y su recepción legislativa en Latinoamérica.
Nos indica que, el teletrabajo es el futuro impuesto de 2020 manera obligatoria, con indicadores sumamente preocupantes y con brechas sociales y tecnológicas alarmantes, pasando desde la falta de empatía entre el empleador y el colaborador, hasta la recarga laboral, impuesta por el empleador hacia el colaborador.

URI:

$h t t p: / / c a m i n o s+2020 \& h l=e s \& a s \_s d t=0 \& a s \_v i s=1 \& o i$

\section{Perticará}

En el contexto y el marco generado a razón de la pandemia

Título:Vulnerabilidad y teletrabajo durante la pandemia. del coronavirus, los trabajadores más vulnerables son aquellos que sufrirán las peores consecuencias de dichas crisis; es momento entonces que los gobiernos y empleadores valoren entre el riesgo de sus exposiciones; y sus remuneraciones y beneficios, pasando por la posibilidad de implementar las condiciones debidas, para la realización del teletrabajo, ofreciendo nuevas oportunidades a dichos colaboradores vulnerables.

URI: https://doi.org/10.11565/oe.vi144.365 


\section{CONCLUSIONES}

En la revisión general de autores e investigadores sobre el teletrabajo, el 50\% de estudios o aportes revisados, coincide en conceptualizar que el teletrabajo es una actividad laboral, a distancia, remota, fuera de oficina y expresiones similares, que es posible realizarlos valiéndose de los diversos recursos y medios tecnológicos de la información y la comunicación (TIC's)

El 20\% de los autores e investigadores revisados, se concentra y coincide en que, para la realización del teletrabajo, es sumamente necesario que el colaborador y el empleador estén totalmente de acuerdo en todos los aspectos del desempeño laboral a distancia; es decir, afirman que el teletrabajo sólo puede darse bajo una absoluta empatía entre ambos. Un 30\% de los autores e investigadores revisados, se concentran y coinciden que el tema del teletrabajo, es un asunto que necesita de urgente regulación y precisión en todos sus aspectos, formas y componentes; en el caso del Perú, este proceso de regulación ya se inició mediante la Ley $\mathrm{N}^{\circ} 30036$ y otros decretos supremos complementarios.

En la revisión específica de artículos científicos, el 100\% de los estudios revisados se concentra y coincide en que el teletrabajo, en el contexto global en el que vivimos actualmente, es una oportunidad que podemos aprovechar si tenemos las competencias y el expertis del dominio de las TIC's; el cual significaría, optimización del tiempo, mejor uso de la energía, cuidado del medio ambiente y tiempo para la familia.

Manifestamos nuestra conformidad, con la gran mayoría de encuestados a nivel nacional, los cuales, no reciben con mucho entusiasmo el tema del teletrabajo, dado a las carencias propias de nuestra realidad; y que muy por el contrario, el teletrabajo en nuestro país, tendría un cierto carácter impositivo.

\section{REFERENCIAS BIBLIOGRAFICAS}

American Psychological Association. (2020). Guía resumen del estilo APA, 75. URL https://apastyle.apa.org/style-grammar-guidelines/index

Caminos, J. (2020). Algunos interrogantes surgidos a raíz del teletrabajo producto de la pandemia COVID-19. Revista de estudio de Derecho Laboral Y Derecho Procesal Laboral Universidad Blas Pascal, (2), 59-70. URL. https://revistas.ubp.edu.ar/index.php/derecho-laboral/article/view/207 
Cano, R., Castro, R., \& Peñafiel, E. (2017). Ventajas y desventajas del teletrabajo aplicados en la ciudad de Lima- Perú, 2016. Repositorio Institucional - UPA, 101. URL: http://repositorio.ulasamericas.edu.pe/handle/upa/156

Cañadas, F. (2017). Teletrabajo: Revisión teórica y análisis jurídico-laboral (pp. 1-231). URL http://digibug.ugr.es/handle/10481/48771

Cataño, L. (2014). El concepto de Teletrabajo: aspectos para la seguridad y salud en el Empleo. CES Salud Pública, 5(1), 82-91. URL https://doi.org/10.21615/2772

Catunta, A. (2018). Aspectos regulatorios del teletrabajo en el Perú: análisis y perspectivas. IUS Revista Pp, 12 (41), 226.

Centro de Estudio del Trabajo y Factores Humanos. (2020). Guía para el teletrabajo en condiciones de pandemia Covid-19, 8. URL https://kinesiologia.uv.cl/images/inicio/destacados/2020/guia-para-elteletrabajo-en-condiciones-de-pandemia-covid-19/documento.pdf

Cifuentes, D. (2020). Teletrabajo: el problema de la institucionalización. Aibi Revista de Investigación, Administración e Ingeniería, 12-20. URL https://doi.org/10.15649/2346030x.749

Diario El Peruano, Normas Legales-496509 (2013) Ley que regula el teletrabajo $\mathrm{N}^{\circ}$ 30036 URL https://busquedas.elperuano.pe/normaslegales/ley-que-regula-elteletrabajo-ley-n-30036-946195-3/

Gabini, S. (2016). Trabajo flexible: conceptualización. Scielo, 46 (118), 25.

Hernández, J. (2019). Editorial a petición del equipo editorial de la Revista de Psicología del Deporte. Revista de Psicologia Del Deporte. Sociedad Revista de Psicologia del Deporte.

Orellana, S., \& Chávez, A. (2014). Suma de Negocios, 5(11), 117-125.

López, D. (2017) El ABC del teletrabajo, 58 (12), 7250-7257. URL https://doi.org/10.1128/AAC.03728-14

López, E., \& Neira, L. (2019). El teletrabajo y el estrés laboral en los colaboradores del banco de crédito del Perú de la provincia de Trujillo. Repositorio Institucional UPAO.

Ministerio de Trabajo y Promoción del Empleo (MTPE) Resolución Ministerial 1222015-TR URL https://busquedas.elperuano.pe/normaslegales/crean-el-modulo- 
de-informacion-y-orientacion-sobre-teletrab-resolucion-ministerial-n-122-2015tr-1243460-2/

Montalvo, J. (2020). Teletrabajo: modalidad en expansión. Enfoques Jurídicos, 0(2), 121-133. URL https://doi.org/10.25009/ej.v0i2.2550

OEFA. (2020) Manual de teletrabajo. Organismo de evaluación y fiscalización ambiental URL https://www.oefa.gob.pe/publicaciones/manual-de-teletrabajo-oefa/

Páez, E., Camargo, S., \& Fonseca, J. (2016). Evaluación del uso de herramientas sincrónicas y asincrónicas en procesos de formación de las ciencias $\begin{array}{llll}\text { agropecuarias. Ciencia } & \text { y } & \text { Agricultura, 13(1), } & \text { URL }\end{array}$ https://doi.org/10.19053/01228420.4808

Perticará, M. y Tejada, M. (2020). Sobre vulnerabilidad y teletrabajo durante la pandemia. Observatorio $\quad$ Económico, $\quad$ 4-5. URL https://doi.org/10.11565/oe.vi144.365

Saco, R. (2006) El teletrabajo: acciones e investigaciones sociales. Revista PUCP (8), 201. URL https://doi.org/10.26754/ojs_ais/ais.19988157

Sánchez, R. (2012). El teletrabajo como tendencia del mercado laboral. RETOS. Revista de Ciencias de la Administración y Economía, 2(4), 143-156. URL http://www.redalyc.org/articulo.oa?id=504550954002

Sanguinetti, W. (2017). Teletrabajo y globalización: en busca de respuestas al desafío de la transnacionalización del empleo (págs. 1-109). URL https://wilfredosanguineti.files.wordpress.com/2009/08/teletrabajo-yglobalizacion-final-informe.pdf

Vidal, D. (2018). Séverine Durin, yo trabajo en casa. Trabajo del hogar de planta, género y etnicidad en Monterrey, México, publicaciones de la casa chata, 2017, 416 p. Problemes d'Amérique Latine, $109(2), \quad 135 . \quad$ URL https://doi.org/10.3917/pal.109.0135 